\title{
Colon Ganglioneuroma
}

National Cancer Institute

\section{Source}

National Cancer Institute. Colon Ganglioneuroma. NCI Thesaurus. Code C7042.

A gang lioneuroma that arises from the colon. It usually presents as a small mucosal polyp. 